\title{
The reported use and effectiveness of Hypericum (St John's wort) on affective symptoms in a depression self-help group
}

\author{
Ruth Dyson ${ }^{\mathrm{a}}$, David S. Baldwin ${ }^{\mathrm{a}}$, Andrew G. Mayers ${ }^{\mathrm{a}}$, \\ Anthony Tiernan ${ }^{\mathrm{b}}$ and Gillian Jenkins ${ }^{\mathrm{c}}$ \\ aCommunity Clinical Sciences, School of Medicine, University of \\ Southampton, Southampton, UK \\ ${ }^{b}$ Depression Alliance, London, UK \\ 'St John's Wort Information Centre, Brackley, UK
}

Correspondence to Andrew Mayers, University Department of Psychiatry, University of Southampton, Royal South Hants Hospital, Brintons Terrace, Southampton SO14 0YG, UK Keywords: Complimentary medicine, Depression, Hypericum, St John's wort

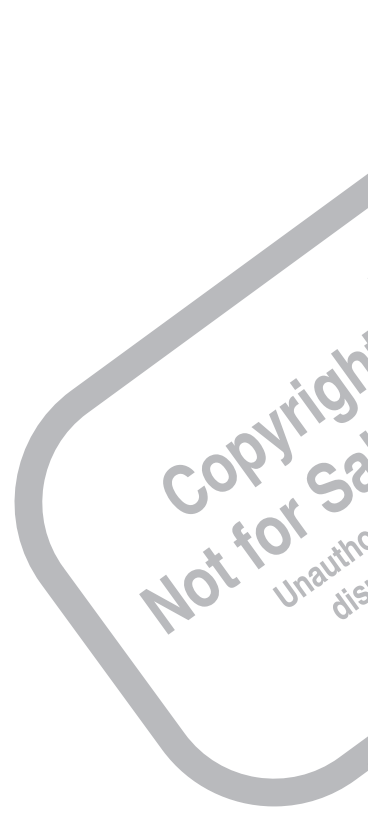

A BSTRACT

A recent meta-analysis suggested that Hypericum perforatum (St John's wort) is an effective treatment for mild to moderate depression and may have a superior side-effect profile to some antidepressant drugs. The aim of this study was to assess the use of herbal remedies in treating depressive and anxiety symptoms, as reported by members of the UK self-help organization Depression Alliance using self-completed questionnaires. More than $50 \%$ of the 452 respondents reported using Hypericum, onequarter of whom also reported concurrent use of traditional antidepressants. Most of the sample reported sufficient symptoms for warranting a diagnosis of major depression, with the majority also describing symptoms suggestive of co-morbid psychiatric conditions. One-half of the Hypericum users experienced symptom improvement, which for most occurred within the first 4 weeks of use. Response was better for those with mild as compared to severe symptoms and poorer for those taking Hypericum alongside other antidepressants. The responders were generally older than non-responders. Adverse effects were reported by one-quarter of users and were mostly psychological in nature. This retrospective survey indicated that use of herbal remedies was common in this population. Although often helpful in relieving symptoms, particularly in those with mild depression, there is a risk of adverse events and drug interactions.

\section{Introduction}

Depressive disorders are a significant cause of morbidity. The lifetime prevalence of major depressive disorder is approximately $20 \%$ for women and is approximately half that for men. ${ }^{1}$ Antidepressants are the most common treatment, although psychological interventions such as cognitivebehavioural therapy and (rarely) electroconvulsive therapy are also an option. Antidepressants are an effective treatment in moderate to severe depression with approximately $70 \%$ of patients responding within 3 months. ${ }^{1}$ Unfortunately side-effects are also common, which can reduce compliance and life quality. Consequently, an effective agent with fewer side-effects than conventional drugs would be beneficial for patients, particularly those with milder symptoms.

Hypericum has been used as a herbal remedy for centuries for its wound-healing, anti-viral, anti-inflammatory, sedative and antidepressant 
properties. However, recent interest has focused primarily on its use as an antidepressant. The use of Hypericum is increasing, along with other complimentary and alternative medicines, ${ }^{2,3} \mathrm{~A}$ recent UK population study reported a lifetime use of $46.6 \%$ for complimentary approaches, ${ }^{4}$ although individuals over the age of 65 years were found to be less likely to use such remedies and women were more likely to use them than men. ${ }^{4}$

Hypericum has a complex pharmacology, mimicking the effects of many classes of antidepressants ${ }^{5}$ including the inhibition of reuptake of serotonin (5-HT), noradrenaline, dopamine, GABA (g-aminobutyric acid) and glutamate. Hypericum also appears to be an inhibitor of cytokine expression, in particular IL-6 (interleukin-6), which may be over-expressed in depression. ${ }^{5}$

Studies have shown Hypericum to be an effective treatment for depression with approximately $60-70 \%$ of patients responding to treatment, ${ }^{6,7}$ particularly in mild depression. Linde et al. ${ }^{6}$ concluded that Hypericum extract was significantly more effective than placebo and similarly effective to conventional antidepressants. However, in a recent study Hypericum was found to be no more efficacious than placebo in moderate to severe or severe depression. ${ }^{8}$ This may have been due to the poor assay sensitivity of that study as sertraline also failed to differentiate from placebo. In addition, many treatment studies have been criticized for using low doses of conventional medication and for inconsistent classification of depressive disorders. Further data are required regarding Hypericum use in different patient populations, such as those with severe depression or those with symptoms not fulfilling the criteria for major depression.

A number of side-effects have been noted with Hypericum, although serious adverse events may be relatively rare compared to the rates of usage. Nevertheless, studies have shown interactions between Hypericum and conventional antidepressants. ${ }^{9,10}$ Hypericum may be superior to conventional medications in its incidence of side-effects or adverse drug reactions. ${ }^{11}$ One metaanalysis $^{6}$ found side-effects in $19.8 \%$ of cases, while a systematic review ${ }^{12}$ reported a range of $12-38 \%$. The sideeffects of Hypericum are generally mild to moderate in intensity and include temporary gastrointestinal disturbance, restlessness, dizziness, fatigue and a dry mouth. ${ }^{7,12,13}$ Allergic reactions are rare and photosensitivity is very rare at therapeutic doses. ${ }^{12}$ However, the lack of data on long-term safety and the possibility of drug interactions mean that Hypericum should be used with care.

Most trials assessing the efficacy of Hypericum have been conducted on restricted subsets of patients, usually with diagnoses of major depressive disorder. However, there are many individuals who do not fulfil the diagnostic criteria for this disorder, but who still experience significant distress and are likely to use Hypericum for relieving their symptoms. Consequently, the participants in this study were recruited from among the membership of the UK self-help organization Depression Alliance. The first part of the investigation was a retrospective study using questionnaires for investigating the use of herbal remedies for depressive and anxiety symptoms. It was predicted that the reported treatment response to Hypericum would decrease with increased depression severity and greater duration of symptoms. It was also expected that the reported response would be poorer in those taking other antidepressants alongside Hypericum and for those who reported co-morbid psychiatric conditions. A further aim of the study was to examine the effect of age and gender on the Hypericum treatment response. This has received little attention in the literature.

The second part, a prospective study using the Hospital Anxiety and Depression Scale and Clinical Global Improvement Scale, is currently being conducted and aims to indicate the effectiveness and tolerability of a Hypericum preparation in a study group that is possibly more representative of Hypericum users.

\section{Methods}

\section{Participants}

The sample consisted of 452 volunteers who were recruited from the membership of Depression Alliance (UK). The mean age of the respondents was 46.9 years and $72.7 \%$ were women. Southampton and South West Hants Local Research Ethics Committee
(S39/00) granted ethical approval in accordance with the principles of the Declaration of Helsinki.

\section{Materials}

A questionnaire designed by one of the authors (R.D.) was used for obtaining information from the participants regarding their previous and current use of herbal remedies, the duration and severity of their depressive symptoms, the extent of co-morbid conditions and their responses to Hypericum along with side-effects and adverse events associated with Hypericum use.

\section{Procedure}

Questionnaires were sent by post to the complete membership ( $n=2700$ ) of Depression Alliance from whom 452 completed forms were received. These were completed by the participants and returned to the researchers. Confidentiality was assured.

\section{Results}

Since the participants were all members of Depression Alliance, they all considered themselves to be depressed, although there was no guarantee how many of them had received a formal psychiatric diagnosis. Their mean symptom duration was 13.9 years.

Three hundred and sixty-five (80.8\%) of the 452 respondents were currently taking medication and 227 (50.2\%) were taking a traditional antidepressant. Ninety-two (40.5\%) participants reported taking selective serotonin reuptake inhibitors, 55 (24.2\%) were taking tricyclic antidepressants and 80 (35.3\%) participants reported taking 'other' antidepressants. Overall 302 participants $(66.8 \%)$ reported taking some form of herbal remedy, 229 $(75.8 \%)$ of whom reported taking Hypericum. Sixty-six participants (28.8\%) reported combining Hypericum and an antidepressant. Overall 191 (63.2\%) of those taking herbal remedies such as Hypericum said that they used the preparation for 'depression', while 42 (13.9\%) mentioned depression/anxiety and 25 (8.3\%) stated that they used Hypericum for anxiety alone. Little effect was 
found for age with respect to Hypericum use, but those over 65 years of age were significantly less likely to use Hypericum than those under 65 years $\left(\chi^{2}=4.096\right.$ and $\left.P<0.05\right)$. There was no effect of gender.

Two hundred and seventy-seven (91.7\%) of the 302 herbal remedy users experienced symptoms that might receive a DSM-IV (Diagnostic and Statistical Manual of Mental Disorders, fourth edition) diagnosis of major depressive disorder. Most of the respondents (252) also reported comorbid problems or conditions alongside depression (83.4\%), 207 (68.5\%) of whom reported anxiety/panic disorder, $61(20.2 \%)$ an eating disorder and 35 (11.6\%) substance abuse. Only 240 participants reported the degree of their depression severity: 44 participants (18.3\%) reported 'mild' symptoms, 119 (49.6\%) reported 'moderate' symptoms and 77 (32.1\%) reported 'severe' symptoms.

Of the respondents describing the effect of Hypericum on their symptoms, 119 (51.9\%) experienced improvement, 92 (40.2\%) described their symptoms as unchanged and 18 (7.9\%) described a worsening of their symptoms. Of those reporting improvement, 91 participants (76.4\%) reported that this occurred within 4 weeks and 57 (47.9\%) between 2 and 4 weeks. The response was significantly better in those participants with mild as compared to severe symptoms $\left(\chi^{2}=12.32\right.$ and $P<0.05$ ). However, there was no effect on response with respect to symptom duration. Response was poorer though for those taking Hypericum with an antidepressant as opposed to Hypericum alone $\left(\chi^{2}=\right.$ 13.16 and $P<0.05)$. Symptom co-morbidity had no effect on response to treatment and there was no effect of gender. In respect of age, the responders were generally older than nonresponders (mean age 49.3 versus 45.8 years $)(P<0.05)$.

Sixty-three participants $(27.5 \%$ of users) reported adverse effects of Hypericum: of these 24 (10.4\%) reported psychological symptoms, ten (4.3\%) described headaches and dizziness, nine (3.9\%) mentioned allergic reactions (itching, rashes), eight (3.5\%) referred to gastrointestinal problems, seven $(3.1 \%)$ reported general aches and pains and five (2.1\%) reported visual disturbances. These side-effects remitted within 1 week for 31 participants (50\%) and within 1 month for 50 of them $(79.5 \%)$.

\section{Discussion}

The use of herbal remedies was common in this sample and at $66.8 \%$ is much higher than the UK average use of these remedies (31.4\%). ${ }^{4}$ Members of a self-help organization may be precisely the kind of individuals who are more likely to use complimentary approaches than those in more traditional settings. Hypericum was considerably more popular than other types of remedy reported by these participants, which may reflect the considerable media attention that this compound currently receives.

The overall response rate to Hypericum of $51.9 \%$ by users, while of a similar magnitude, was marginally lower than those rates previously reported (60-70\%)., 6 This may reflect the inclusion here of participants who would be excluded from randomized controlled trials, for example due to severe or chronic depression or use of antidepressant medication. As predicted, response was significantly better for those with milder as compared to more severe symptoms. This supports findings in the literature that Hypericum treatment is effective in those with mild-moderate disease but less so in severe disease. ${ }^{6-8}$ However, the expected effect for symptom duration against the response rate was not found. As predicted, response was poorer for those taking Hypericum alongside a traditional antidepressant as compared to those taking the medication alone. However, against expectations there was no effect for symptom co-morbidity on the response rates. These issues warrant further research since little has been reported about this relationship.

The study revealed an age-related effect on both the use of herbal remedies and response to Hypericum treatment. Those over 65 years were less likely to use herbal remedies. This finding was supported by a recent study, which also found lower rates of complementary and alternative medicine (CAM) use in the over 65 years age group. ${ }^{4}$ In respect to response rates and age, the current study found that the responders were significantly older than non-responders. Research has found that women have a significantly higher use of herbal remedies, ${ }^{4}$ but this was not replicated here. This may be because gender did not affect use in the current study population or it could have been due to the low percentage of male respondents. Furthermore, the current study found no effect of gender on treatment response. Again, further research is warranted in this respect.

A key finding in the current study was that $7.9 \%$ of Hypericum users described a worsening of symptoms and $27.5 \%$ experienced one or more adverse events and, thus, Hypericum treatment is not without potential drawbacks. A recent meta-analysis ${ }^{6}$ quoted a $19.8 \%$ incidence of sideeffects and a systematic review ${ }^{12}$ found incidences ranging from 12 to $38 \%$. The incidence here was similar to these figures, as was the nature of the sideeffects, although more studies could be conducted on psychological disturbances associated with use.

Nevertheless, the level of adverse effects remains a cause for concern, particularly as more participants obtained information on taking Hypericum from the media than they did from health professionals. This is disturbing for two reasons: Hypericum users may not be receiving adequate information on the safety of using the preparation and general practitioners may be unaware that their patients are taking Hypericum when they prescribe other treatments. This was compounded by the finding here that $28.8 \%$ of the Hypericum users also took a traditional antidepressant, thereby risking drug interactions such as serotonin syndrome. ${ }^{9}$ While this is unlikely, a possible case has been reported in the literature. $^{10}$

\section{Summary and implications}

These results suggest that patients with mild or a low number of symptoms may respond well to Hypericum irrespective of symptom duration. Thus, Hypericum may be a reasonable alternative to conventional antidepressants in these patients, particularly if they experience troublesome side-effects 
with these medications. Due to the high number of people taking herbal remedies and the risk of associated adverse events or drug interactions, it is important that doctors are fully aware of what preparations their patients are taking.

Further studies are required in order to examine the reliability and validity of the measures used here. Furthermore, whilst the application of self-completed questionnaires is an efficient method of data collection, little information was available on formal diagnoses or on the dosage of Hypericum taken. Nevertheless, useful preliminary information for assessing the merit of Hypericum in people with mild to moderate depression is presented in the data provided by this study.

\section{Collaborating organizations}

Depression Alliance is the UK's leading charity working for people affected by depression. It coordinates self-help groups, provides support and information, campaigns in order to raise awareness and conducts research into the incidence, experience and treatment of depression. The organization has 20 full-time staff and 2700 members.

The St John's Wort Information Centre is an independent organization providing information and advice about Hypericum and conducting research into its use, efficacy and tolerability.

\section{References}

1. Baldwin DS, Hirschfeld RMA. Fast FactsDepression. Oxford: Health Press; 2001.

2. Owen DK, Lewith G, Stephens CR. Can doctors respond to patients' increasing interest in complementary and alternative medicine? BMJ 2001; 322:154-158.

3. Vincent C, Furnham A. Complementary medicine: state of the evidence. $J R$ Soc Med 1999; 92:170-177.

4. Thomas KJ, Nicholl JP, Coleman P. Use and expenditure on complementary medicine in England: a population based survey. Complement Ther Med 2001; 9:2-11.

5. Nathan PJ. Hypericum perforatum (St John's wort): a non-selective reuptake inhibitor? A review of the recent advances in its pharmacology. J Psychopharmacol 2001; 15:47-54.
6. Linde K, Ramirez G, Mulrow CD, Pauls A, Weidenhammer W, Melchart D. St John's wort for depression - an overview and meta analysis of randomised clinical trials. BMJ 1996; 313:253-258.

7. Hippius H. St John's wort (Hypericum perforatum) - a herbal antidepressant. Curr Med Res Opin 1998; 14:171-184.

8. Hypericum Depression Trial Study Group. Effect of Hypericum perforatum (St John's wort) in major depressive disorder: a randomized controlled trial. $J \mathrm{Am}$ Med Assoc 2002; 287:1807-1814.

9. Gordon JB. Selective serotonin reuptake inhibitors and St John's wort: possible toxicity? Am Family Phys 1998; 57:950.

10. Wheatly D. St John's wort: more knowledge needed. Pharm J 2000; 265 (7104):49.

11. Gaster B, Holroyd J. St John's wort for depression: a systematic review. Arch Intern Med 2002; 160:152-156.

12. Ernst E, Rand JI, Barnes J, Stevinson C. Adverse effects of the herbal antidepressant St. John's wort (Hypericum perforatum L.). Eur J Clin Pharmacol 1998; 54:589-594.

13. Medicines Control Agency. Reminder: St John's wort (Hypericum perforatum) interactions. Curr Problems Pharmacovigil 2000; 26:6-7. 